\title{
Lethality in PARP-1/Ku80 Double Mutant Mice Reveals Physiological Synergy During Early Embryogenesis
}

\author{
Melinda S. Henrie ${ }^{1,2}$, Akihiro Kurimasa ${ }^{1}$, Sandeep Burma ${ }^{1}$, \\ Josiane Ménissier-de Murcia ${ }^{3}$, Gilbert de Murcia ${ }^{3}$, Gloria C. $\mathrm{Li}^{4}$, and David J. Chen ${ }^{1 *}$ \\ Lawrence Berkeley National Laboratory \\ Life Sciences Division \\ 1 Cyclotron Road, Berkeley, CA 94720
}

Tel: 510-495-2861; Fax: 510-486-6816; E.mail: djchen@lbl.gov

${ }^{1}$ Lawrence Berkeley National Laboratory, Berkeley, CA

${ }^{2}$ University of New Mexico School of Medicine, Albuquerque, NM

${ }^{3}$ Ecole Superieure de Biotechnologie de Strasbourg, CNRS, 67400 Illkirch-

Graffenstaden, France

${ }^{4}$ Memorial Sloan-Kettering Cancer Center, New York, NY

*Corresponding author

Key words: PARP-1; Ku80; Homologous repair; Base excision repair; embryonic lethality 


\section{Abstract}

$\mathrm{Ku}$ is an abundant heterodimeric nuclear protein, consisting of $70-\mathrm{kDa}$ and $86-$ $\mathrm{kDa}$ tightly associated subunits that comprise the DNA binding component of DNAdependent protein kinase. Poly(ADP)ribose polymerase-1 (PARP-1) is a 113-kDa protein that catalyzes the synthesis of poly(ADP-ribose) on target proteins. Both $\mathrm{Ku}$ and PARP-1 recognize and bind to DNA ends. $\mathrm{Ku}$ functions in the non-homologous end joining (NHEJ) repair pathway whereas PARP-1 functions in the single strand break repair and base excision repair (BER) pathways. Recent studies have revealed that PARP-1 and Ku80 interact in vitro. To determine whether the association of PARP-1 and Ku80 has any physiological significance or synergistic function in vivo, mice lacking both PARP-1 and Ku80 were generated. The resulting offspring died during embryonic development displaying abnormalities around the gastrulation stage. In addition, PARP1-/-Ku80-/- cultured blastocysts had an increased level of apoptosis. These data suggest that the functions of both Ku80 and PARP-1 are essential for normal embryogenesis and that a loss of genomic integrity leading to cell death through apoptosis is likely the cause of the embryonic lethality observed in these mice.

\section{Introduction}


Poly(ADP)ribose polymerase-1 (PARP-1) is a nuclear zinc-finger protein that is the founding member of a family of polymerases with roles in preserving genomic integrity in multi-cellular organisms [1-4]. PARP-1 is a 113-kDa multimodular protein consisting of a 46-kDa DNA binding N-terminal domain, a 22-kDa central automodification domain bearing a BRCT motif, and a 54-kDa catalytic C-terminal domain [5]. The 46-kDa N-terminal recognizes DNA breaks in a sequence-independent manner. Upon binding DNA, PARP-1 becomes catalytically active, using NAD+ to synthesize chains of poly(ADP-ribose) on a limited number of acceptor proteins, including itself. Automodification of PARP-1 results in electrostatic repulsion of itself from DNA, causing its dissociation from DNA and subsequent deactivation [1]. In vitro, PARP-1 has been shown to be a binding partner for XRCC1 through yeast-two hybrid screens [6]. PARP-1 has also been shown to play a role in base excision repair $[7,8]$.

PARP-1-/- mice are viable and show no overtly abnormal phenotypes; however, exogenous stress by alkylating agents and $\gamma$-irradiation leads to a severe increase in mortality compared to controls [9-11]. Furthermore, the absence of PARP-1 is associated with an increase in sister chromatid exchanges (SCEs), micronuclei, and chromosome breaks that mirror spontaneous genomic stress $[10,12,13]$.

The DNA dependent protein kinase (DNA-PK) holoenzyme is formed by the association of a large kinase subunit (DNA-PKcs) and a DNA binding heterodimer of 
$\mathrm{Ku} 70 / 80$ proteins $[14,15] . \mathrm{Ku}$ is a phosphoprotein that binds with high affinity to DNA double strand breaks (DSBs). Atomic force microscopy studies have shown that Ku binds DNA internally as well as at the ends, and can form DNA loops by self-association [16]. Upon generation of a DSB, Ku binds to the broken DNA ends and subsequently recruits DNA-PKcs and DNA ligase IV/ XRCC4 complex to the sites of damage $[17,18]$. This may play a role in aligning DNA termini and may help to facilitate end re-joining.

Ku80-/- mice are severely growth deficient, are defective in V(D)J [variable (diversity) joining] recombination, and are ionizing radiation (IR)-sensitive [19-21]. Kudeficient fibroblasts have a prolonged doubling time due to loss of proliferating cells and exhibit premature senescence and an increase in telomere fusions $[19,21]$. The phenotype of Ku80-deficient mice indicates that $\mathrm{Ku} 80$ is essential for lymphogenesis, protection from ionizing radiation, and growth regulation [19].

An accumulation of evidence suggests that PARP-1 and Ku80 interact in several cellular pathways. PARP-1 and Ku80 co-immunoprecipitate from mammalian cell extracts [22-24]. In addition, a novel role for PARP-1 and Ku80 has been suggested by their ability to co-localize to matrix attachment sequences [25]. This suggests a possible role in the maintenance of chromatin structure and perhaps transcription regulation. In vitro, PARP-1 and Ku80 can simultaneously occupy the same DNA end and cooperatively stimulate DNA ligase IV, the human ligase implicated in DNA DSB repair (S. M. Yannone and D.J. Chen; unpublished observations). PARP-1 can poly (ADP- 
ribosyl)ate DNA-PKcs in vitro which enhances its kinase function [22]. PARP-1 also appears to cooperate with DNA-PKcs in vivo to suppress homologous recombination in mouse cells [23].

Recent studies indicate that Ku and PARP-1 may have overlapping roles in the repair of DNA damage and the maintenance of genomic integrity. In order to ascertain the functional relationship between PARP-1 and Ku80, and to determine if a synergy exists between these two proteins, mice deficient in both PARP-1 and Ku80 were generated. 


\section{Materials and Methods}

\subsection{Generation of PARP-1/Ku80 mice}

PARP-1 null mice [10] were mated to Ku80 [19] null or hemizygous mice. Mice are in a mixed C57BL6 X 129SVJX NIH Swiss Black background. PARP-1+/-/Ku80+/mice were intercrossed to generate PARP-1-/-Ku80+/- mice which were bred to produce PARP-1-/-Ku80-/- embryos.

\subsection{Genotyping of mice and embryos}

The PARP-1 genotype was determined by Southern blot analysis [10]. Total genomic DNA extracted from tails was digested with EcoRI and transferred overnight to a Hybond-N+ nylon membrane (Amersham Pharmacia Biotech). The membrane was hybridized using a PARP-1 specific probe. Hybridized products were detected by autoradiography.

The Ku80 genotype was determined using PCR as previously described [19]. Primer K1 (5' ATTGTGATGTGTGGGACACG 3') detects both wild type and mutant alleles, K2 (5' AGCTTCCACCCTCTAGAGAT3') detects only the wild type allele and K3 (5' TAAAGCGCATGCTCCAGACT3') detects only the mutant allele. After 2 min 
incubation at $94^{\circ} \mathrm{C}$, the alleles were amplified using the following conditions: $20 \mathrm{sec}$ at $94^{\circ} \mathrm{C}, 20 \mathrm{sec}$ at $58^{\circ} \mathrm{C}$ and $1 \mathrm{~min}$ at $72^{\circ} \mathrm{C}$ for 32 cycles, followed by a 10 min extension cycle. PCR products were run on $1.2 \%$ agarose $0.5 \mathrm{X}$ TBE gel and stained with ethidium bromide.

Embryos and blastocysts were obtained from intercrosses of Ku80+/-mice bred on a PARP-1 null background. To screen for the Ku80 genotype, DNA was prepared from embryos and blastocysts by incubating in a lysis buffer containing $10 \mathrm{mM}$ Tris- $\mathrm{HCl} \mathrm{pH}$ 8.0, $50 \mathrm{mM} \mathrm{KCl}, 1.5 \mathrm{mM} \mathrm{MgCl}, 20.001 \%$ gelatin, $0.45 \% \mathrm{NP}-40$, and $0.45 \%$ proteinase $\mathrm{K}$. Samples were incubated at $56^{\circ} \mathrm{C}$ for 4 hours followed by $90^{\circ} \mathrm{C}$ for 30 minutes. The total DNA isolate was amplified using the same PCR conditions described above.

PCR products were then analyzed by Southern blotting using a ${ }^{32} \mathrm{P}$-end labeled internal oligonucleotide probe K10 (5' TATGGGTGCCTGGAGCTAGAAT 3'). Hybridized products were detected by autoradiography.

\subsection{Mouse embryo analysis}

Whole embryos were observed and photographed under a dissecting microscope or low powered microscope. Embryonic stage was estimated using timed matings. 


\subsection{Blastocyst culture and TUNEL analysis}

E3.5 blastocysts were collected from natural matings between PARP-1-/-Ku80+/or PARP-1+/+/Ku80+/- intercrosses, and rinsed in Dulbecco's $\mathrm{Mg} / \mathrm{Ca}$-free phosphate buffered saline (Gibco). Blastocysts were cultured in 4-well chamber slides in $1 \mathrm{ml}$ Knockout ${ }^{\mathrm{TM}}$ Dulbecco's modified Eagle medium (Gibco) supplemented with $2 \mathrm{mM}$ Lglutamine, 1X non-essential amino acids, 1,000U/ml leukemia inhibitory factor (LIF), 100 units $/ \mathrm{ml}$ penicillin, $100 \mu \mathrm{g} / \mathrm{ml}$ streptomycin, $1 \%$ fungizone, $0.1 \mathrm{mM}$ beta mercaptoethanol, and $20 \%$ fetal bovine serum. Cells were maintained at $37^{\circ} \mathrm{C}$ in a humidified atmosphere of $5 \% \mathrm{CO}_{2}$. Blastocysts were fixed in $4 \%$ paraformaldehyde after 4 or 5 days in culture. Permeabilization and TUNEL analysis were performed using the Apoalert DNA fragmentation assay (Clontech) according to manufacturer's instructions with the following modifications: a hydrophobic ring was drawn around the blastocyst and the assay was performed using micro-volumes of reagents applied directly to the cells to prevent dissociation of the sample. Images were taken at 200X magnification and captured using the Spot Diagnostic imaging system. 


\section{Results}

\subsection{PARP-1 and Ku80 are both required for normal embryonic development}

Intercrosses of PARP-1-/-Ku80+/- mice were expected to yield double-knockout (PARP-1-/-Ku80-/-) offspring with a Mendelian Frequency of 0.25 . However, genotyping of 72 live-born pups identified no double-knockout mice (Table 1), suggesting that the loss of both proteins leads to a defect during embryogenesis. To determine the stage at which the double-knockouts stopped developing, embryos from PARP-1-/-Ku80+/- intercrosses were analyzed (Table 2 and Figure 1). At the preimplantation stage (E3.5), an approximately Mendelian proportion of embryos were double-knockouts (12 out of 42 embryos examined). All E3.5 embryos appeared normal regardless of their genotype indicating that the defect leading to lethality of the doubleknockout embryos occurred after implantation. Double-knockout embryos were also detected from E6.5 to E9.5. However, these embryos were severely growth retarded and abnormal, or appeared as yolk sacs surrounding a disorganized mass of cells undergoing resorbtion. By E6.5, varying degrees of developmental arrest occurred in the double mutants. Some embryos were more developed (Figure1, Panel b) than other embryos at the same stage (Panel c). Examples of normal developing embryos are shown in Figure 1, (a,f,g) along with abnormal (b, c) and resorbed (e,h,i) conceptuses at E9.5, E11.5 and E13.5 respectively. We were unable to genotype embryos undergoing maternal 
resorbtion. Between day 11.5 -14.5 p.c., empty decidua (Fig.1, e) but no abnormal embryos were observed, suggesting that any double null embryos had been resorbed at an earlier stage. Empty decidua were also found prior to E11.5. Also, we were unable to generate any mouse embryonic fibroblasts (MEFs) from the E6.5-E9.5 double-knockout embryos. These data suggest that PARP-1 and Ku80 play an essential role early in mouse embryogenesis and that the loss of both proteins has a lethal effect on the early post-implantation embryo at a stage as early as the onset of gastrulation (E6.5).

\subsection{PARP-1-/-Ku80-/- embryos die by apoptosis around the time of gastrulation}

Because the defect in cellular proliferation in PARP-1-/-Ku80-/- mice first appeared at some point between E3.5 and E6.5, blastocysts were cultured and screened for the presence of apoptosis. Terminal deoxynucleotidyl transferase-mediated dUTP nick end labeling (TUNEL) staining revealed that after 4-5 days in culture, the inner cell mass (ICM), but not the trophoblastic giant cells (TGC) of PARP-1-/-Ku80-/- cultures

exhibited a high degree of DNA fragmentation indicative of apoptosis (Fig. 2, a). In contrast, PARP-1-/-/Ku80+/+ (Fig. 2, b), PARP-1+/+Ku80-/- (Fig. 2, c), and PARP1+/+Ku80+/+ (Fig. 2, d) cells exhibited background levels of TUNEL staining. Blastocysts were co-stained with DAPI for nuclear detection. The level of apoptosis in 
double-knockout blastocysts analyzed at day 3 was comparable to background levels found in PARP-1 single-knockout blastocysts at that stage (data not shown) suggesting that PARP-1 and Ku80 are both required for the proliferation of blastocyst cells after 3 days in culture. 


\section{Disscussion}

\subsection{Early embryonic lethality in PARP-1-/-Ku80-/- mice}

Breeding experiments were carried out to generate mice deficient in both $\mathrm{Ku} 80$ and PARP-1. Most combinations of PARP-1 and Ku80 offspring, including PARP-1+//Ku80+/- and PARP-1-/-/Ku80+/- mice, appeared normal and healthy and were fertile. Careful analysis of over 72 offspring revealed no surviving PARP-1-/-Ku80-/- mice. This implied that there was a defect during embryogenesis resulting from the loss of both proteins. To determine when the double-knockouts were dying, embryos from PARP-1-//Ku80+/- intercrosses were analyzed at different stages of development. No doubleknockout embryos were detected at mid-gestation (E11.5-14.5), though empty decidua were found, indicating the embryos were dying at an earlier stage. Double-knockout embryos were detected from E6.5 to E9.5, however these embryos, even at E6.5, were severely growth retarded and abnormal or appeared as yolk sacs surrounding a disorganized mass of cells undergoing resorbtion. Empty decidua were also found at these earlier stages. E3.5 embryos appeared normal regardless of their genotype. These data suggest that PARP-1 and Ku80 play an essential role in early embryogenesis in mice and that the loss of both proteins has an effect on the embryo at a stage as early as the onset of gastrulation (E6.5). 
A recent study has revealed that mouse embryos undergoing gastrulation are hypersensitive to low doses of ionizing radiation [26]. The consequence of this type of damage is the initiation of p53-dependant apoptosis in embryonic, but not in extraembryonic cells. Embryonic cells give rise to critical lineages, whereas extraembryonic cells are temporary and divide at a much slower rate, which may explain why they can tolerate damage. This hypersensitivity to DNA damage was specific to gastrulation and was not observed at any other developmental stage. This suggests that gastrulation may be a period in development when the mouse may be extremely sensitive to DNA damage, and that cells undergo apoptosis rather than risk the production of defective cells that may contribute to critical lineages. PARP-1-/-Ku80-/- mice appear to be lethal as early as E6.5 which corresponds to the onset of gastrulation. Interestingly, early embryonic lethality has also been observed at the gastrulation stage for certain knockout mice of the BER (XRCC1, and AP-endonuclease) and HR (Brca1 Brca2, Rad50, Rad51) DNA repair pathways [27-36]. In addition, mouse knockouts of ataxia telangiectasia related protein (ATR), an ATM/rad3-related protein which regulates cell cycle checkpoints in response to IR and ultraviolet radiation and which may regulate Brca1 and Brca2, are found to be embryonic lethal around the time of gastrulation as well [37]. Thus, the loss of certain components of the BER and HR repair pathways leads to embryonic lethality at or immediately before gastrulation, perhaps as a result of unrepaired DNA damage, or due to a defect in DNA-damage signaling. Furthermore, a 
PARP-1 deficiency combined with an ataxia telangiectasia mutated (ATM) [38] or Ku80 deficiency (this study) and DNAPKcs/ATM deficiency [39] is lethal at the same stage, indicating that disrupting specific sets of two DNA repair pathways is incompatible with normal gastrulation.

A double mutant for PARP-1/DNA-PKcs has been generated [23]. These mice are viable though growth retarded. They show a partial rescue of the severe combined immunodefieciency (SCID) phenotype, presumably due to an increase in recombinational events. The fact that PARP-1/DNA-PKcs double-knockout mice survive, whereas PARP-1/Ku80 double-knockout mice die in early embryogenesis, further supports the hypothesis that DNA-PKcs and Ku have distinct, as well as overlapping roles in the cell. Interestingly, the phenotype of the PARP-1/Ku80 double-knockout mouse closely resembles the phenotype of the PARP-1/ATM double knockout mouse [38].

4.2. PARP-1 and Ku80 may function together to guard the embryo against genomic insult.

The inability of PARP-1-/-Ku80-/- embryos to repair endogenous DNA damage in early development could account for the embryonic lethal phenotype of these mice. Gastrulation is a period of high cell division rate [26]. A direct result of the increased 
metabolism in cells at gastrulation would theoretically correlate with a marked increase in reactive oxygen species which cause oxidative damage to cells. In the absence of both PARP-1 and Ku80, both SSB and DSB repair may be impaired, weakening the cell's defense against endogenous DNA damage and increasing the likelihood of lethal chromosomal breaks and replication failure. Cells suffering from DNA damage may be eliminated by apoptosis as is observed by us in the cultured double-knockout blastocysts. This has also been suggested as an explanation for the lethality observed in PARP-1 and ATM double mutant mice [38].

In conclusion, the early embryonic lethality observed in PARP-1 and Ku80 double-KO mice can perhaps be explained by an increase in genomic instability resulting in cell death through apoptosis. The phenotype of these mice is more severe than either of the single knockouts, indicating that either PARP-1 and Ku80 function in distinct pathways, or that their functions are, perhaps, redundant is some aspects, such that the loss of either one can be tolerated, but not both. Determining the exact mechanism(s) underlying the lethality of PARP-1 and Ku80 knockouts will be the subject of future studies.

\section{Acknowledgments}


We would like to thank S.M. Yannone for sharing unpublished data, Eric Campeau and Mark Brenneman for critical reading of this manuscript, and Esther Koai, Jeanna Park, and Pei Chen for their excellent assistance with the mouse colony. This work was funded by US Dept. of Energy and NIH grants AG17709 and CA50519 to D.J.C. and by Commissariat à l'Energie Atomique, Electricité de France and Association pour la Recherche contre le Cancer to G.d.M.

\section{References}


[1] G. de Murcia and J. Menissier de Murcia Poly(ADP-ribose) polymerase: a molecular nick-sensor, Trends Biochem Sci 19 (1994) 172-176.

[2] P.A. Jeggo DNA repair: PARP - another guardian angel?, Curr Biol 8 (1998) R49-51.

[3] T. Lindahl, M.S. Satoh, G.G. Poirier and A. Klungland Post-translational modification of poly(ADP-ribose) polymerase induced by DNA strand breaks, Trends Biochem Sci 20 (1995) 405-411.

[4] S. Smith The world according to PARP, Trends Biochem Sci 26 (2001) 174-179.

[5] G. de Murcia, V. Schreiber, M. Molinete, B. Saulier, O. Poch, M. Masson, C. Niedergang and J. Menissier de Murcia Structure and function of poly(ADPribose) polymerase, Mol Cell Biochem 138 (1994) 15-24.

[6] M. Masson, C. Niedergang, V. Schreiber, S. Muller, J. Menissier-de Murcia and G. de Murcia XRCC1 is specifically associated with poly(ADP-ribose) polymerase and negatively regulates its activity following DNA damage, Mol Cell Biol 18 (1998) 3563-3571.

[7] F. Dantzer, G. de La Rubia, J. Menissier-De Murcia, Z. Hostomsky, G. de Murcia and V. Schreiber Base excision repair is impaired in mammalian cells lacking Poly(ADP- ribose) polymerase-1, Biochemistry 39 (2000) 7559-7569.

[8] L.O. Prasad R, Kim SJ, Kedar P, Yang XP, Vande Berg BJ, Wilson SH. 
DNA polymerase beta -mediated long patch base excision repair. Poly(ADPribose)polymerase-1 stimulates strand displacement DNA synthesis., J Biol Chem 276 (2001) 32411-32414.

[9] M. Masutani, T. Nozaki, E. Nishiyama, T. Shimokawa, Y. Tachi, H. Suzuki, H. Nakagama, K. Wakabayashi and T. Sugimura Function of poly(ADP-ribose) polymerase in response to DNA damage: gene- disruption study in mice, Mol Cell Biochem 193 (1999) 149-152.

[10] J.M. de Murcia, C. Niedergang, C. Trucco, M. Ricoul, B. Dutrillaux, M. Mark, F.J. Oliver, M. Masson, A. Dierich, M. LeMeur, C. Walztinger, P. Chambon and G. de Murcia Requirement of poly(ADP-ribose) polymerase in recovery from DNA damage in mice and in cells, Proc Natl Acad Sci U S A 94 (1997) 73037307.

[11] S. Shall and G. de Murcia Poly(ADP-ribose) polymerase-1: what have we learned from the deficient mouse model?, Mutat Res 460 (2000) 1-15.

[12] Z.Q. Wang, L. Stingl, C. Morrison, M. Jantsch, M. Los, K. Schulze-Osthoff and E.F. Wagner PARP is important for genomic stability but dispensable in apoptosis, Genes Dev 11 (1997) 2347-2358.

[13] C.M. Simbulan-Rosenthal, B.R. Haddad, D.S. Rosenthal, Z. Weaver, A. Coleman, R. Luo, H.M. Young, Z.Q. Wang, T. Ried and M.E. Smulson Chromosomal aberrations in PARP(-/-) mice: genome stabilization in immortalized cells by 
reintroduction of poly(ADP-ribose) polymerase cDNA, Proc Natl Acad Sci U S A 96 (1999) 13191-13196.

[14] A. Dvir, S.R. Peterson, M.W. Knuth, H. Lu and W.S. Dynan Ku autoantigen is the regulatory component of a template-associated protein kinase that phosphorylates RNA polymerase II, Proc Natl Acad Sci U S A 89 (1992) 1192011924.

[15] T.M. Gottlieb and S.P. Jackson The DNA-dependent protein kinase: requirement for DNA ends and association with Ku antigen, Cell 72 (1993) 131-142.

[16] R.B. Cary, S.R. Peterson, J. Wang, D.G. Bear, E.M. Bradbury and D.J. Chen DNA looping by $\mathrm{Ku}$ and the DNA-dependent protein kinase, Proc Natl Acad Sci U S A 94 (1997) 4267-4272.

[17] S.E. Critchlow and S.P. Jackson DNA end-joining: from yeast to man, Trends Biochem Sci 23 (1998) 394-398.

[18] D.A. Ramsden and M. Gellert $\mathrm{Ku}$ protein stimulates DNA end joining by mammalian DNA ligases: a direct role for $\mathrm{Ku}$ in repair of DNA double-strand breaks, Embo J 17 (1998) 609-614.

[19] A. Nussenzweig, C. Chen, V. da Costa Soares, M. Sanchez, K. Sokol, M.C. Nussenzweig and G.C. Li Requirement for Ku80 in growth and immunoglobulin V(D)J recombination, Nature 382 (1996) 551-555. 
[20] H. Ouyang, A. Nussenzweig, A. Kurimasa, V.C. Soares, X. Li, C. Cordon-Cardo, W. Li, N. Cheong, M. Nussenzweig, G. Iliakis, D.J. Chen and G.C. Li Ku70 is required for DNA repair but not for $\mathrm{T}$ cell antigen receptor gene recombination In vivo, J Exp Med 186 (1997) 921-929.

[21] Y. Gu, K.J. Seidl, G.A. Rathbun, C. Zhu, J.P. Manis, N. van der Stoep, L. Davidson, H.L. Cheng, J.M. Sekiguchi, K. Frank, P. Stanhope-Baker, M.S. Schlissel, D.B. Roth and F.W. Alt Growth retardation and leaky SCID phenotype of Ku70-deficient mice, Immunity 7 (1997) 653-665.

[22] T. Ruscetti, B.E. Lehnert, J. Halbrook, H. Le Trong, M.F. Hoekstra, D.J. Chen and S.R. Peterson Stimulation of the DNA-dependent protein kinase by poly(ADP-ribose) polymerase, J Biol Chem 273 (1998) 14461-14467.

[23] C. Morrison, G.C. Smith, L. Stingl, S.P. Jackson, E.F. Wagner and Z.Q. Wang Genetic interaction between PARP and DNA-PK in V(D)J recombination and tumorigenesis, Nat Genet 17 (1997) 479-482.

[24] S. Galande and T. Kohwi-Shigematsu Caught in the act: binding of Ku and PARP to MARs reveals novel aspects of their functional interaction, Crit Rev Eukaryot Gene Expr 10 (2000) 63-72.

[25] S. Galande and T. Kohwi-Shigematsu Poly(ADP-ribose) polymerase and Ku autoantigen form a complex and synergistically bind to matrix attachment sequences, J Biol Chem 274 (1999) 20521-20528. 
[26] B.S. Heyer, A. MacAuley, O. Behrendtsen and Z. Werb Hypersensitivity to DNA damage leads to increased apoptosis during early mouse development, Genes Dev 14 (2000) 2072-2084.

[27] D.L. Ludwig, M.A. MacInnes, Y. Takiguchi, P.E. Purtymun, M. Henrie, M. Flannery, J. Meneses, R.A. Pedersen and D.J. Chen A murine AP-endonuclease gene-targeted deficiency with post- implantation embryonic progression and ionizing radiation sensitivity, Mutat Res 409 (1998) 17-29.

[28] R.S. Tebbs, M.L. Flannery, J.J. Meneses, A. Hartmann, J.D. Tucker, L.H. Thompson, J.E. Cleaver and R.A. Pedersen Requirement for the Xrcc1 DNA base excision repair gene during early mouse development, Dev Biol 208 (1999) 513529.

[29] H. Gu, J.D. Marth, P.C. Orban, H. Mossmann and K. Rajewsky Deletion of a DNA polymerase beta gene segment in $\mathrm{T}$ cells using cell type-specific gene targeting, Science 265 (1994) 103-106.

[30] T. Tsuzuki, Y. Fujii, K. Sakumi, Y. Tominaga, K. Nakao, M. Sekiguchi, A. Matsushiro, Y. Yoshimura and MoritaT Targeted disruption of the Rad51 gene leads to lethality in embryonic mice, Proc Natl Acad Sci U S A 93 (1996) 62366240.

[31] G. Luo, M.S. Yao, C.F. Bender, M. Mills, A.R. Bladl, A. Bradley and J.H. Petrini Disruption of mRad50 causes embryonic stem cell lethality, abnormal embryonic 
development, and sensitivity to ionizing radiation, Proc Natl Acad Sci U S A 96 (1999) 7376-7381.

[32] S.X. Shen, Z. Weaver, X. Xu, C. Li, M. Weinstein, L. Chen, X.Y. Guan, T. Ried and C.X. Deng A targeted disruption of the murine Brcal gene causes gammairradiation hypersensitivity and genetic instability, Oncogene 17 (1998) 31153124.

[33] C.Y. Liu, A. Flesken-Nikitin, S. Li, Y. Zeng and W.H. Lee Inactivation of the mouse Brcal gene leads to failure in the morphogenesis of the egg cylinder in early postimplantation development, Genes Dev 10 (1996) 1835-1843.

[34] T. Ludwig, D.L. Chapman, V.E. Papaioannou and A. Efstratiadis Targeted mutations of breast cancer susceptibility gene homologs in mice: lethal phenotypes of Brca1, Brca2, Brca1/Brca2, Brca1/p53, and Brca2/p53 nullizygous embryos, Genes Dev 11 (1997) 1226-1241.

[35] V.L. Cressman, D.C. Backlund, A.V. Avrutskaya, S.A. Leadon, V. Godfrey and B.H. Koller Growth retardation, DNA repair defects, and lack of spermatogenesis in BRCA1-deficient mice, Mol Cell Biol 19 (1999) 7061-7075.

[36] S.K. Sharan, M. Morimatsu, U. Albrecht, D.S. Lim, E. Regel, C. Dinh, A. Sands, G. Eichele, P. Hasty and A. Bradley Embryonic lethality and radiation hypersensitivity mediated by Rad51 in mice lacking Brca2, Nature 386 (1997) 804-810. 
[37] E.J. Brown and D. Baltimore ATR disruption leads to chromosomal fragmentation and early embryonic lethality, Genes Dev 14 (2000) 397-402.

[38] J.M. Murcia, M. Mark, O. Wendling, A. Wynshaw-Boris and G. de Murcia Early Embryonic Lethality in PARP-1 Atm Double-Mutant Mice Suggests a Functional Synergy in Cell Proliferation during Development, Mol Cell Biol 21 (2001) 18281832.

[39] K.E. Gurley and C.J. Kemp Synthetic lethality between mutation in Atm and DNA-PK(cs) during murine embryogenesis, Curr Biol 11 (2001) 191-194. 


\section{Figure Legends}

Figure 1. Whole mount embryos at different stages of development, E9.5 (a-c), E11.5 (d-f), and E13.5 (g-i), derived from intercrosses of PARP-1-/-Ku80+/- mice. Wild type (a) and PARP-1-/-Ku80-/- (b, c) conceptuses showing varying degrees of abnormality. E11.5 (d) shows normal decidua (d, right) and abnormal decidua (d, left). Abnormal decidua shown above in (d) is cut in half (e) and is shown to be empty. When the normal decidua is dissected, the embryo and the placenta are found (f). E13.5 (g-i) normal (g) and two small abnormal decidua (h, i). Scale bar, $1.0 \mathrm{~mm}$. (a-i)

Figure 2. TUNEL analysis of blastocysts after 5 days in culture. Embryos were harvested at E3.5, grown in culture for 5 days, stained with fluorescein (TUNEL) and DAPI and photographed using phase contrast microscopy. PARP-1-/-Ku80-/- blastocysts (a) exhibit an increased level of apoptosis compared to PARP-1-/-/Ku80+/+, PARP1+/+/Ku80-/-, and PARP-1+/+/Ku80 +/+ blastocysts (b-d). Arrows point to the inner cell mass (ICM) and the trophoblastic giant cells (TGC).

\section{Table 1}


Genotypes of 24 Day Old Progeny Derived From

PARP-1-/-Ku80+/- Crosses

\begin{tabular}{llc} 
PARP-1 & Ku80 & observed (expected) \\
\hline & & \\
$-/-$ & $+/+$ & $30(18)$ \\
$-/-$ & +- & $42(36)$ \\
$-/-$ & $-/-$ & $0(18)$
\end{tabular}

total offspring: 72 


\section{Table 2}

Genotypes of Embryos and Blastocysts Derived from PARP-1-/-Ku80+/Crosses

PARP-1-/-

Days p.c.

$11.5-14.5(24)$

4

9

0

11

0

$9.5(12)$

2

4

4*

2

0

$8.5(10)$

4

3

$1 *$

1

1

$7.5(8)$

1

1

0

2

4

$6.5(9)$

1

3

$1 *$

3

1

blastocysts

$3.5(42)$

14

14

12

NA

2

total analysed: 105

$\mathrm{ND}=$ genotype data not available

$\mathrm{NA}=$ not applicable

* denotes abnormal and severely growth retarded embryos 
Figure 1
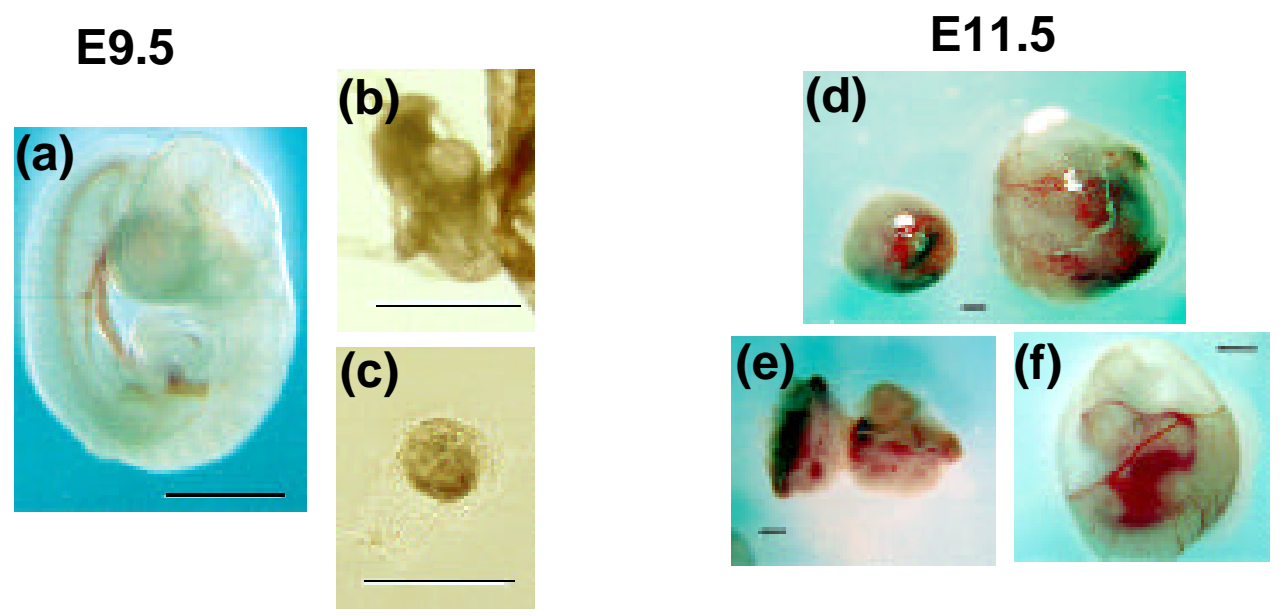

\section{E13.5}
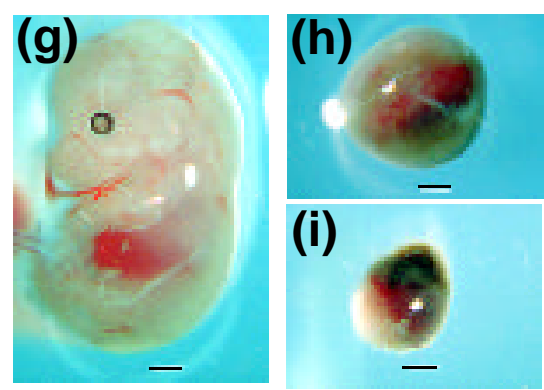
Figure 6. Whole mount embryos at different stages of development, E9.5 (A-C), E11.5 (D-F), and E13.5 (G-I), derived from intercrosses of PARP-/-/Ku80+/mice. Wildtype (A) and abnormal (B,C) PARP-/-/Ku80-/- conceptuses showing a varying degree of severity. Normal decidua (D, right)and abnormal decidua (D, left). Abnormal decidua shown above (D) is cut in half $(E)$ and is shown to be empty. Embryo (F) dissected out of normal decicual (D)shown in placenta is normal. E13.5 (G-I) normal $(G)$ and two small abnormal decidua $(\mathrm{H}, \mathrm{I})$. Scale bar, $1.0 \mathrm{~mm}$. (A-I) 
Figure 2

PARP/Ku80

20X

(a)

$$
-|-|-\mid-
$$

(b)

$$
-/-/+/+
$$

(c)

$$
+/+/-/-
$$

(d)
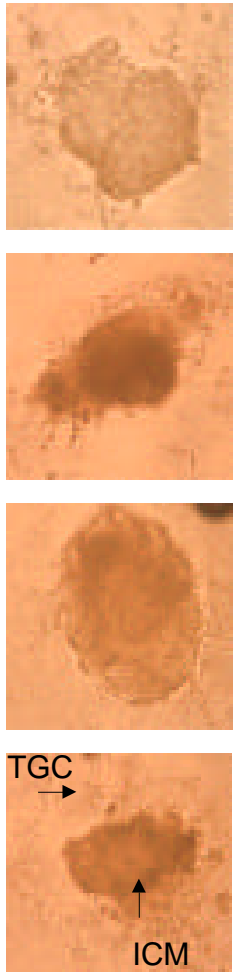

TUNEL DAPI
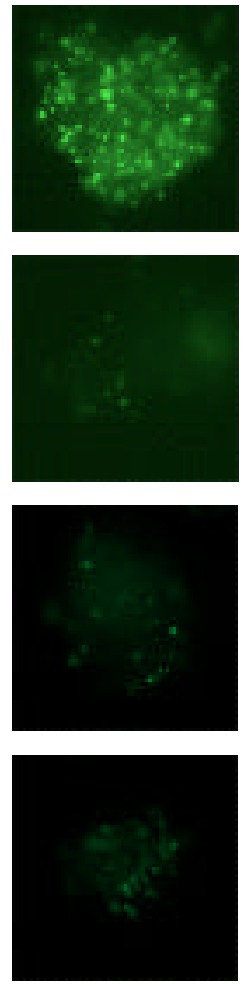
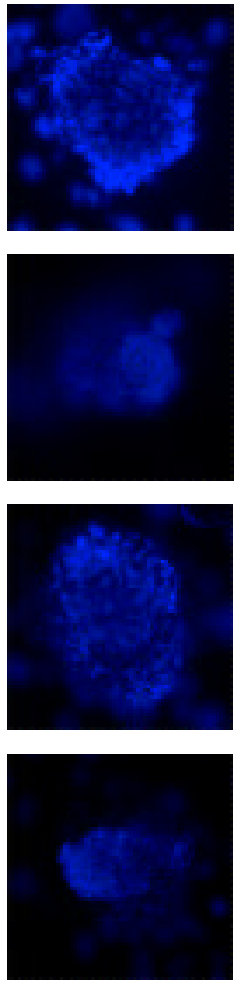
Figure 8. TUNEL analysis of blastocysts after 5 days in culture. PARP-/-/Ku80 -/- blastocysts (A) exhibit an increased level of apoptosis compared to PARP-/-/Ku80. 
Figure 8. TUNEL analysis of blastocysts after 5 days in culture. PARP-/-/Ku80 -/- blastocysts (A) exhibit an increased level of TUNEL apoptosis compared to PARP-///Ku80+/+, PARP+/+/Ku80-/-, and PARP+/+/Ku80 +/+ blastocysts (B-D). 
